François Höpflinger, Stefanie Spahni \& Pasqualina Perrig-Chiello

\title{
Persönliche Bilanzierung der Herausforderungen einer Verwitwung im Zeit- und Geschlechtervergleich
}

\author{
Subjectively perceived challenges of widowhood - Gender differences and \\ time effects
}

\begin{abstract}
Zusammenfassung:
Bisherige Forschung hat die Verwitwung entweder primär als soziales oder als individuelles Ereignis untersucht, selten jedoch wurden beide Perspektiven verbunden. Zudem ist wenig darüber bekannt, inwiefern bisherige Forschungsergebnisse Perioden- oder Kohorteneffekte wiederspiegeln. In diesem Beitrag wird die persönliche Bilanzierung nach der Verwitwung älterer Schweizer Frauen und Männer im Geschlechterund Zeitvergleich untersucht ${ }^{1}$. Die Datenbasis beruht auf Befragungen von 1.197 verwitweten Frauen und Männern (Alter: 65-102 Jahre), welche 1979, 1994 und 2011 durchgeführt wurden. Während sich die wirtschaftlichen und sozialen Rahmenbedingungen nach einer Verwitwung namentlich bei Frauen - im Zeitvergleich verbessert haben, zeigen sich bezüglich psychischer Herausforderungen einer Verwitwung keine periodenspezifischen Veränderungen. Psychisch bleibt der Partnerverlust auch bei günstigen Sozialbedingungen ein kritisches Lebensereignis, das individualisiert bewältigt werden muss.
\end{abstract}

Schlagworte: Verwitwung, psychosoziale Herausforderungen, Geschlechterunterschiede, Zeiteffekte

\begin{abstract}
:
Research on bereavement has traditionally focussed on widowhood as either a social or individual event, but rarely under both perspectives. Furthermore, little is known whether existing research results mirror period or cohort effects. The aim of this article is to investigate constancies and changes in the retrospective perception of the challenges of widowhood of elderly women and men living in different decades in Switzerland. Data stem from three questionnaire studies with 1.197 widowed men and women (aged 65-102 years) carried out in 1979, 1994, and 2011. Results reveal that the subjective interpretations mirror the significant improvement of the economic and social situation of widowed individuals in Switzerland over the last decades, particularly for women. In contrast, no significant time effects can be observed with regard to the psychological challenges of marital loss in old age (redefinition of sense of life, feelings of loneliness). These findings suggest, that even in good socio-economic conditions widowhood remains psychologically a critical life event.
\end{abstract}

Key words: Widowhood, psychosocial challenges, gender differences, time effects

1 Vom Schweizerischen Nationalfonds gefördertes Projekt (CRSII1_129922) sowie mit Unterstützung von Pro Senectute Schweiz, unter der Leitung von Michel Oris, Universität Genf, und Pasqualina Perrig-Chiello, Universität Bern, sowie weiteren Gesuchstellenden. 


\section{Einleitung - Verwitwung als kritisches Lebensereignis}

Verwitwung bzw. Partnerverlust durch Tod ist vor allem im höheren Lebensalter ein häufiges Ereignis, welches aufgrund geschlechtsspezifischer Unterschiede von Lebenserwartung und Partnerschaftsverhalten Frauen häufiger trifft als Männer (Frauen ehelichen häufig einen älteren Partner) (Bennett 2008; Carr 2004). Partnerverlust durch Tod bedeutet oft das Ende einer langjährigen Partnerbeziehung, mit allen persönlichen und familialen Konsequenzen, welche den Schluss einer engen und intimen Lebens- und Haushaltsgemeinschaft für den überlebenden Partner beinhaltet. Entsprechend gilt Verwitwung als bedeutsames kritisches Lebensereignis mit negativen psychischen und sozialen Folgen. Vor allem die erste Zeit nach einer Verwitwung ist durch eine erhöhte Anfälligkeit für körperliche und psychische Erkrankungen gekennzeichnet (Jin/Chrisatakis 2009; Schaan 2009). Die Bewältigung einer Verwitwung hängt längerfristig sowohl von den Umständen des Partnerverlustes als auch von den sozialen und psychischen Ressourcen des überlebenden Partners oder der überlebenden Partnerin ab (Stroebe et al. 2007). „Eines der häufigsten Befunde der Trauerforschung ist, dass Personen, die zuvor an psychischen Störungen litten (z.B. klinische Depression oder Angststörungen), mit hoher Wahrscheinlichkeit auch im Umgang mit einem Verlust Schwierigkeiten erleben. Folglich gelten vorangegangene psychische Probleme als erheblicher Risikofaktor. Dasselbe gilt für Verluste, die unter gewaltsamen und unerwarteten Bedingungen stattfinden.“ (Boerner 2012: 233). Die Variabilität der Reaktionen auf einen Partnerverlust ist allerdings ausgeprägt und die Auswirkungen eines Partnerverlustes können von Depressivität, Verzweiflung und Suizidalität bis hin zu persönlichem Wachstum und erfolgreicher Adaptation an eine neue Lebenssituation reichen (vgl. Itzhar-Naborro/Smoski 2012).

Verwitwung ist oft mit einer Reduktion der sozialen Beziehungen, Netzwerke und des sozialen Engagements verbunden, vor allem unmittelbar nach einem Partnerverlust (Bennett 2005; Hollstein 2002). Allerdings reduziert ein dichtes außerfamiliales Netzwerk vor dem Partnerverlust das Risiko einer sozialen Isolation nach der Verwitwung, wie in einer Sekundäranalyse des deutschen Alterssurvey 2002 sichtbar wurde (Schmid 2010). Eine französische Längsschnittstudie verdeutlicht in einem Vergleich von verwitweten und verheirateten Personen im Alter zwischen 62 und 75 Jahren ebenfalls, dass Verwitwete auf sozialen Indikatoren negativere Werte aufzeigen als gleichaltrige Verheiratete: Sie weisen eine negativere Einstellung zum Leben und zur Pensionierung auf, leiden häufiger unter Einsamkeit und depressiven Tendenzen. Zudem sind sie weniger oft in Freizeitaktivitäten engagiert und häufiger sozial isoliert (Delbès/Gaymu 2002). Auch wirtschaftlich kann Verwitwung negative Auswirkungen aufweisen. Trotz Ausbau des Wohlfahrtsstaates ist Verwitwung in Europa - wie Daten der SHARE-Studie verdeutlichen - weiterhin für manche Betroffene mit einem substanziellen Rückgang der absoluten wie relativen Einkommensposition verbunden (Gorlé/van den Bosch 2008: 303). Allerdings ist der Einkommens- und Rentenverlust nach einem Partnerverlust stark von den sozialpolitischen Rahmenbedingungen (wie Höhe der Hinterlassenenrenten) eines Landes abhängig (vgl. Burkauser et al. 2005).

Im Allgemeinen wird davon ausgegangen, dass Männer eine Verwitwung schlechter bewältigen als Frauen und beispielsweise häufiger mit Suizidalität und Depressivität auf den Verlust reagieren (Stroebe et al. 2001; van Grootheest et al. 1999; Perrig-Chiello/ Hutchison 2010). Dies scheint auch für eine Verwitwung im hohen Lebensalter zu gelten 
(Berg 2006; Lee et al. 2001). Insofern Männer auch in der nachberuflichen Lebensphase sozial und emotional stärker auf eine Paarbeziehung ausgerichtet sind als Frauen, scheint Partnerverlust bei Männern im Alter verstärkt zu Einsamkeitsgefühlen beizutragen (Dykstra/de Jong-Gierveld 2004; Pinquart 2003). Geschlechtsspezifische Unterschiede des psychologischen Befindens nach einer Verwitwung verwischen sich allerdings stark bis vollständig, wenn andere bedeutsame Variablen, wie etwa vorheriges Wohlbefinden und Alter bei Verwitwung, mitkontrolliert werden (vgl. Bennett 2005; Lee/DeMaris 2001) oder longitudinale Verläufe untersucht wurden (Lee/DeMaris 2007).

\section{Verwitwung - individuelles oder soziales Schicksal - zwei alternative Ansätze}

Die bisherige Verwitwungsforschung ist dadurch charakterisiert, „dass die (zumeist kurzund mittelfristigen) psychischen Konsequenzen der Verwitwung sehr breit untersucht werden, die längerfristigen, und insbesondere die sozialen Folgen jedoch noch weitgehend unklar sind." (Hollstein 2002: 64). Was die sozialen Folgen und die psychische Bewältigung des kritischen Lebensereignisses ,Verwitwung' betrifft, lassen sich - vereinfacht dargestellt - zwei alternative Interpretationsansätze gegenüberstellen:

Ein erster Ansatz betrachtet Partnerverlust als primär individuelles Lebensereignis, welches Personen in allen Gesellschaften in analoger Weise (negativ) berührt. Nach diesem Ansatz sind primär psychische Faktoren für Bewältigung und Bilanzierung einer Verwitwung bedeutsam (Bennett 2008; Boerner 2012); dies namentlich in modernen Gesellschaften, wo verwitweten Frauen und Männern kein sozialer Sonderstatus zugebilligt wird und Verwitwung ein sozial wenig sichtbares individuelles Schicksal darstellt.

Ein zweiter, alternativer Ansatz interpretiert Partnerverlust auch als soziales Ereignis, welches - wie andere soziale und familiale Lebensereignisse - durch gesellschaftliche Rahmenbedingungen und familiale Strukturen beeinflusst wird, und dies auch in individualisierten Gesellschaften (Hollstein 2002).

Analog hierzu wurde die Notwendigkeit einer Unterscheidung zwischen Verwitwung (widowhood) als primär soziales Phänomen und Todesfall bzw. Verlust des Partners (bereavement) als primär individuelles, psychologisches Phänomen auch von Bennett und Soulsby (2012) nahegelegt, um die Folgen besser einordnen zu können.

Trifft der erste Interpretationsansatz zu, sollten sich im persönlichen Erleben und in der subjektiven Bilanzierung einer Verwitwung im historischen Zeitvergleich keine markanten Veränderungen ergeben, wogegen der zweite Ansatz davon ausgeht, dass auch Verwitwung bedeutsamen Perioden- und Generationeneffekten unterliegt. Nach Ansicht von Hollstein (2002) steht zu erwarten, dass jüngere Generationen von Frauen und Männern ,insgesamt über bessere individuelle Ressourcen verfügen, sich nach der Verwitwung ein befriedigendes ,neues Leben“ aufzubauen.“ (ibd.: 326-327).

Dank gleichlautenden Fragen im Rahmen dreier Erhebungen aus der französischsprachigen Schweiz ist es uns in diesem Beitrag in einzigartiger Weise möglich, Items zur persönlichen Bilanzierung einer Verwitwung - im Sinne einer subjektiven retrospektiven Gesamteinschätzung der sozialen und psychischen Herausforderungen des Partnerverlusts - in einem längeren Zeitvergleich (Erhebungszeitpunkte: 1979, 1994 und 2011) zu analysieren. Dadurch lässt sich überprüfen, inwiefern sich die persönliche Bilanzierung einer 
Verwitwung in den letzten dreißig Jahren wesentlich verändert hat, oder ob umgekehrt Verwitwung als sozial wenig sichtbares kritisches Lebensereignis so stark individualisiert bewältigt wird, so dass nur marginale Periodeneffekte auftreten. Unsere Ausgangsthese ist die Annahme, dass die in den letzten Jahrzehnten stattgefundenen wirtschaftlichen und sozialen Verbesserungen in der Lebenslage älterer Frauen und Männer in der Schweiz negative wirtschaftliche und soziale Folgen einer Verwitwung verringert haben, sich dies jedoch auf die psychischen Folgen (wie etwa Gefühle der Vereinsamung) weniger ausgewirkt hat, da es sich um stark individualisierte Erlebnisdimensionen handelt, die unabhängig von gesellschaftlichen Rahmenbedingungen negativ erfahren werden.

Gleichzeitig kann untersucht werden, ob und in welcher Richtung geschlechtsspezifische Unterschiede in der persönlichen Bilanzierung einer Verwitwung auftreten. Der gesellschaftliche Wandel von Ehe und Familie der letzten Jahrzehnte - etwa der Wandel von exklusiven Ehebeziehungen zu offenen Partnerschaften, erhöhte Erwerbstätigkeit von Ehefrauen u.a. - kann möglicherweise zu einer Reduktion oder Auflösung geschlechtsspezifischer Unterschiede in den sozialen Folgen einer Verwitwung geführt haben. Ferner hat in der Schweiz der Ausbau der Altersvorsorge dazu beigetragen, dass sich die Armutsrisiken von Witwern und Witwen angeglichen haben (Wanner/Fall 2011).

Soweit dies von der Datenlage möglich ist, werden zusätzliche Variablen, welche die Bewältigung einer Verwitwung beeinflussen können - wie subjektive Gesundheit, erfahrene soziale Unterstützung, Vorhersehbarkeit des Todes des Partners (unerwartet oder erwartet, z.B. nach langer Krankheit), gegenwärtiges Lebensalter und Dauer seit Partnerverlust - in den Zeitvergleich einbezogen. Es gibt empirische Evidenz, dass der Tod des Partners/der Partnerin negative Auswirkungen auf die physische Gesundheit hat (Stroebe et al. 2007) und diese wiederum mit psychischen Problemen der Betroffenen interkorreliert. Somit wird angenommen, dass das Ausmaß an empfundenen Schwierigkeiten in Folge des Partnerverlusts negativ mit der subjektiven Gesundheit zusammenhängt. Verschiedene Studien zeigen, dass erfahrene soziale Unterstützung durch Verwandte und Freunde die vielfältigen negativen Auswirkungen der Verwitwung abschwächen kann (Ha/Ingersoll-Dayton 2011; Norris/Murrell 1990; Pinquart 2003). Es gibt ferner empirische Evidenz, dass die Vorhersehbarkeit des Todes des Partners/der Partnerin eine Rolle spielt, wobei ein unerwarteter, plötzlicher Tod mit erhöhten Schwierigkeiten im Umgang mit dem Verlust einhergeht (Boerner 2012). So scheinen auch Schwierigkeiten bei der Bewältigung einer Verwitwung im höheren Lebensalter geringer zu sein als bei jüngeren Betroffenen (Verwitwung im Alter als altersnormierte biografische Transition) (Bennett/Soulsby 2012; Bonanno et al. 2004; Perkins/Harris 1990). Im Allgemeinen wird angenommen, dass die Anpassung an die negativen Folgen der Verwitwung mit zunehmender Zeitdauer seit dem Verlust auftritt. Allerdings zeigt der aktuelle Forschungsstand widersprüchliche Ergebnisse hinsichtlich der Dauer des Adaptations-Prozesses und bezüglich der Frage, ob das psychische Wohlbefinden der Verwitweten zum Ursprungswert vor dem Verlust zurückkehrt (Bennet 1997; Clark et al. 2008; Itzhar-Nabarro/Smoski 2012; Koren/Lowenstein 2008; Lucas et al. 2003). 


\section{Methode}

\section{Studiendesign und Stichproben}

Im Rahmen des vom Schweizerischen Nationalfonds geförderten Projektes, Vivre-LebenVivere $^{2}$ wurde 2011/12 in den Kantonen Genf, Wallis, Bern, Basel-Stadt und BaselLandschaft sowie im Tessin eine Erhebung zu den Lebens- und Gesundheitsbedingungen der älteren Bevölkerung (65 Jahre und älter) durchgeführt. Die Stichprobe ist nach Geschlecht, Altersgruppe und Region geschichtet. Die Teilnehmenden stammen aus einer Zufallsstichprobe aus den Bevölkerungsregistern der Kantone bzw. Gemeinden und wurden schriftlich wie auch telefonisch zur Teilnahme an der Studie eingeladen. In den französischsprachigen Regionen (Genf, urbane Region; Zentralwallis, ländliche Region) wurden 1208 Interviews durchgeführt. Analoge Erhebungen mit weitgehend identischen Fragen und gleichen Stichprobenverfahren wurden in denselben Regionen bereits 1979 und 1994 durchgeführt. 1979 wurden 1608 Personen und 19942100 Personen in privaten Haushalten interviewt. Die drei Untersuchungen zu unterschiedlichen Zeitpunkten zielten darauf ab, die zeitliche Entwicklung in der Lebenslage, im Gesundheitszustand und im Aktivitätsniveau älterer Menschen zu erfassen (GUGRISPA 1983; Lalive d'Epinay et al. 2000). Die nachfolgenden Analysen beschränken sich auf die Daten der verwitweten 65jährigen und älteren Personen der Kantone Genf und Wallis, da nur in diesen beiden Regionen die Erhebungen 1979, 1994 und 2011 durchgeführt wurden. ${ }^{3}$ Aus der Befragung 1979 sind es 455 Personen (339 Frauen, 116 Männer) im Alter von 65-92 Jahren (M = 75.60, SD = 6.49), aus jener von 1994431 Personen (318 Frauen, 113 Männer) im Alter von 65-103 Jahren $(\mathrm{M}=77.43, \mathrm{SD}=7.67)$ und aus der Erhebung 2011 sind es 301 Personen (206 Frauen, 95 Männer) im Alter von 65-100 Jahren $(\mathrm{M}=82.23, \mathrm{SD}=7.87)$.

\section{Kontexthintergrund: sozialer Wandel (1979-2011)}

Die Lebenslage älterer Menschen hat sich in den letzten Jahrzehnten stark gewandelt. Zum ersten stieg die Lebenserwartung (ab Geburt) weiter an, zwischen 1978/83 bis 2010 für Männer von 72.4 Jahren auf 80.5 Jahre und für Frauen von 79.1 Jahren auf 84.6 Jahre. Aufgrund der erhöhten Lebenserwartung hat sich auch das durchschnittliche Alter der Witwen und Witwer erhöht, bei den befragten Witwen zwischen 1979 und 2011 von 75.2 Jahren auf 82.1 Jahre und bei den Witwern in der gleichen Zeitperiode von 76.7 auf 82.7 Jahre. Parallel dazu wurde die Altersvorsorge - in der Schweiz basierend auf einem Drei-Säulen Konzept

2 SNF-Projekt (CRSII1_129922) sowie mit Unterstützung von Pro Senectute Schweiz, unter der Leitung von Michel Oris, Universität Genf und Pasqualina Perrig-Chiello, Universität Bern, sowie weiteren Gesuchstellenden.

3 Die Westschweiz übernahm in der Schweiz bezüglich Altersforschung eine Vorreiterrolle und entsprechend wurden in Genf und Wallis Jahrzehnte vor der deutschsprachigen Schweiz systematische Erhebungen zur Lage alter Menschen durchgeführt. Alterspolitisch unterscheiden sich Westschweiz und Deutschschweiz bis heute vor allem darin, dass Altersfragen in der französischsprachigen Schweiz stärker kantonal organisiert sind, wogegen Altersfragen in den deutschsprachigen Kantonen weitgehend in kommunaler Verantwortung liegen. 
(Altersversicherung nach Umlageverfahren, berufliche Vorsorge nach Kapitaldeckungsverfahren und steuerlich begünstigtes privates Sparen) - in den letzten Jahrzehnten stark ausgebaut, mit der Folge, dass sich die Altersarmut reduzierte und die Lebens- und Wohnqualität großer Teile der älteren Bevölkerung deutlich verbesserte (Höpflinger 2009a; Moser 2006). In den letzten Jahrzehnten kam es in der Schweiz - auch wohlstandsbedingt - deshalb zu einer stärkeren Ausdehnung der gesunden Lebenserwartung als in anderen europäischen Ländern (vgl. Jagger et al. 2011). Schon zwischen 1974 und 1994 wurde eine signifikante Verbesserung der gesundheitlichen und psychischen Lage älterer Menschen festgestellt (vgl. Lalive d'Epinay et al. 2000), und dieser Prozess hat sich in den letzten Jahrzehnten weiter verstärkt, was auch zu einer aktiveren Gestaltung der nachberuflichen Lebensphase beigetragen hat (vgl. Höpflinger 2011). Verstärkt wurde der Strukturwandel des Alters einerseits dadurch, dass sich die Bildungsexpansion ab den 1970er Jahren auch bei älteren Personen auswirkte. Entsprechend sank der Anteil der befragten Witwen ohne weiterführende berufliche Ausbildung zwischen 1979 und 2011 von $79 \%$ auf $27 \%$ und bei den befragten Witwern von $68 \%$ auf 22\%. Familial haben - analog wie in Deutschland - in den letzten dreißig Jahren einerseits Verhaltensänderungen hinsichtlich Eheschließung und Scheidung (nachlassende Heiratsneigung, gestiegene Scheidungshäufigkeit) zum Rückgang der Verheirateten- und zur Erhöhung der Geschiedenenquote beigetragen. Andererseits bewirkte die gestiegene Lebenserwartung bzw. die daraus resultierende demografische Alterung der Bevölkerung ebenfalls eine Verschiebung der Anteile der Zivilstände in einzelnen Altersklassen (z.B. einerseits Erhöhung der in einer Ehe verbrachten Lebenszeit, andererseits mehr hochaltrige Personen, die ihren Lebenspartner verloren haben) (Heiniger 2013).

\section{Messinstrumente und Indikatoren}

Für die vorliegende Analyse der persönlichen Bilanzierung einer Verwitwung ist vor allem interessant, dass die genau gleichen sechs Items in allen drei Erhebungen in derselben Form erfragt wurden, was es erlaubt, die entsprechenden Antworten im Zeitverlauf zu vergleichen. So wurden bei verwitweten Personen in allen drei Erhebungen die erlebten Schwierigkeiten nach dem Verlust des Partners erfragt: „Ich werde Ihnen verschiedene Situationen beschreiben, mit denen man nach dem Tod des Partners/der Partnerin konfrontiert werden kann. Inwiefern bereiteten Ihnen diese Schwierigkeiten?" Auswahlmöglichkeiten waren: Finanzielle Probleme; Mangel an sozialen Kontakten; Einsamkeit; Sich daran gewöhnen, alles selber machen zu müssen; Alleine gewisse Verantwortungen zu übernehmen; Dem Leben einen Sinn geben. ${ }^{4}$ Als Antwortkategorien wurden vorgegeben: ,ein schwerwiegendes Problem'; ,ein großes Problem'; ,ein kleineres Problem'; ,kein Problem'. Die Antworten zwischen den einzelnen Bilanzierungsitems sind zu allen drei Erhebungszeitpunkten stark interkorreliert. Ein Summenindex (Skala der subjektiven Bilanzierung empfundener Schwierigkeiten nach der Verwitwung) aller sechs Items ergibt zeitübergreifend eine hohe Konstruktreliabilität (Cronbach's Alpha: 1979: 0.78, 1994: 0.78, 2011: 0.73). Da es sich bei den Antwortkategorien um ordinalskalierte Antwortvorgaben - mit unterschiedlichen sub-

4 Ein siebtes Item „Schwierigkeit, Arbeit zu finden“ wurde für die Analyse verwitweter Personen im Rentenalter $(65+)$ nicht verwendet. 
jektiven Distanzen zwischen zwei Antwortkategorien - handelt, ist bei der Interpretation von Mittelwerten allerdings eine gewisse Zurückhaltung geboten.

Die aktuelle subjektive Gesundheit wurde mittels einer 5-stufigen Skala als ,sehr gut', ,gut', ,befriedigend', ,eher schlecht' oder ,schlecht' eingeschätzt. Die verwitweten Teilnehmenden wurden außerdem gefragt, ob sie auf die Hilfe von jemandem zählen konnten, um sich mit diesen Situationen zurechtzufinden. Die Frage konnte mit ,Ja' oder, Nein ${ }^{6}$ beantwortet werden. Weiter wurde erfragt, ob der Tod des Partners plötzlich und unerwartet - beispielsweise als Folge eines Unfalls, einer kurzen Krankheit - oder nach einer langen Krankheit erfolgte. Das gegenwärtige Lebensalter wurde mit Hilfe des Geburtsdatums der befragten Witwern und Witwen, die Zeitdauer seit der Verwitwung mit Hilfe des Todesjahres des Partners/der Partnerin berechnet.

\section{Resultate}

\section{Subjektive Bilanzierung empfundener Schwierigkeiten bei der Verwitwung nach Zeitperiode und Geschlecht}

Ein erster Vergleich - basierend auf Mittelwerten der Skala ,Bilanzierung empfundener Schwierigkeiten nach der Verwitwung' (vgl. Tabelle 1) - verdeutlicht im Zeitvergleich über mehr als dreißig Jahre eine relativ ausgeprägte Konstanz. Die Mittelwertunterschiede sind im Zeitvergleich statistisch nicht signifikant und auch die Standardabweichungen hier nicht aufgeführt - haben sich nicht systematisch verändert. ${ }^{5}$ Einzig bei den Frauen zeigt sich nach 1994 ein statistisch signifikanter, aber keineswegs ausgeprägter Trend in Richtung einer leicht verbesserten Verwitwungsbilanzierung. Was die geschlechtsspezifischen Unterschiede betrifft, ergeben sich zu keinem Zeitpunkt statistisch signifikante Unterschiede zwischen betroffenen Frauen und Männern. Methodisch weist dies darauf hin, dass die verwendete Skala zur Messung der persönlichen Bilanzierung einer Verwitwung eine periodenübergreifende Reliabilität aufzuweisen scheint, und dass der Summenindex nicht von vornherein einem geschlechterbezogenen Bias unterliegt.

Tabelle 1: Bilanzierung empfundener Schwierigkeiten nach der Verwitwung im Zeitvergleich 1979-2011 (Skalenwerte)

\begin{tabular}{rrrrrrr}
\hline & & Total & & \multicolumn{2}{c}{ Männer } & \multicolumn{2}{c}{ Frauen } \\
& $\mathrm{N}$ & $\mathrm{M}$ & $\mathrm{n}$ & $\mathrm{M}$ & $\mathrm{n}$ & $\mathrm{M}$ \\
\hline 1979 & 442 & 11.1 & 112 & 10.5 & 330 & 11.2 \\
1994 & 416 & 11.3 & 108 & 10.7 & 308 & 11.6 \\
2011 & 293 & 9.9 & 89 & 10.2 & 204 & 9.8 \\
\hline
\end{tabular}

$\mathrm{M}=$ Mittelwerte: additive Summenskala von 6 Items ( $\min .=6$, max. $=24)$ : je höher der Wert, desto negativer die Bilanzierung.

5 Als statistisch signifikant werden im Text Gruppen- bzw. Mittelwertunterschiede und Korrelationen bezeichnet, die gemäß gewähltem Test (F-Test für Mittelwertunterschiede, Chi-Quadrat-Test für Gruppenunterschiede) auf dem 1\%-Niveau von einer statistisch nicht signifikanten Verteilung abweichen. 
Unterschiede nach Geschlecht: in keiner Erhebungsperiode signifikant auf 1\% (F-Test). Unterschiede 1979-1994: nicht signifikant, Unterschiede 1994-2011: nur bei Frauen signifikant auf 1\% (F-Test für zwei unabhängige Stichproben).

Als nächster Schritt werden die Werte der Einzelitems betrachtet, wobei es aufgrund des ordinalen Charakters der Items - mit unterschiedlichen subjektiven Distanzen zwischen Antwortkategorien - sinnvoll ist, nicht von Mittelwerten, sondern von Verteilungswerten auszugehen. Zunächst wird die Gesamtverteilung nach verwitweten Befragten insgesamt angeführt. Dies wird anschließend durch einen systematischen Vergleich nach Geschlecht ergänzt.

Bei zwei von sechs Bilanzierungsitems ergeben sich klare und statistisch signifikante Trends (vgl. Tabelle 2):

Tabelle 2: Erfahrene Probleme nach der Verwitwung: 1979-2011 (Prozentangaben)

\begin{tabular}{lcccccc}
\hline & \multicolumn{3}{c}{ Schwerwiegendes/großes Problem } & \multicolumn{3}{c}{ Kein Problem } \\
& 1979 & 1994 & 2011 & 1979 & 1994 & 2011 \\
\hline Finanzielle Probleme & 29 & 21 & $13^{*}$ & 57 & 61 & 80 \\
Mangel an sozialen Kontakten & 25 & 16 & $15^{*}$ & 59 & 66 & 70 \\
Einsamkeit & 45 & 54 & 38 & 37 & 29 & 39 \\
Alles selber machen zu müssen & 26 & 32 & 26 & 60 & 49 & 57 \\
Allein Verantwortung übernehmen zu müssen & 24 & 30 & 20 & 64 & 54 & 61 \\
Dem Leben Sinn geben & 25 & 28 & 20 & 60 & 45 & 61 \\
\hline
\end{tabular}

N 1979 (je nach Item) = 386-436; N 1994 (je nach Item $)=405-430 ;$ N 2011 (je nach Item $)=$ 294-301.

* Lineare Trendentwicklung bei der Gesamtverteilung zwischen 1979 und 2011 auf 1\% signifikant (ChiQuadrat-Test).

Zum ersten werden finanzielle Probleme einer Verwitwung in den neueren Erhebungen seltener erwähnt. Der Anteil an Verwitweten, die große bis sehr große finanzielle Probleme durch den Partnerverlust erfuhren, sank zwischen 1979 und 2011 von 29\% auf 13\%. Der Anteil derjenigen, die keine finanziellen Probleme anführen, stieg von 57\% auf 80\%. Neben der Tatsache, dass in der Schweiz die Altersvorsorge in den letzten Jahrzehnten ausgebaut wurde, hat die systematische Integration von Witwen- und Witwerrenten in die Altersvorsorge (Alters- und Hinterlassenenvorsorge, AHV) dazu beigetragen, dass Bezügerinnen und Bezüger von Hinterlassenenrenten finanziell oft gut abgesichert sind. In den letzten Jahrzehnten haben zudem mehr Personen vom Ausbau der beruflichen Renten (nach Kapitaldeckungsverfahren) profitiert, und gegenwärtig liegt das Medianeinkommen verwitweter Personen sogar über dem Medianeinkommen nicht verwitweter Personen in vergleichbarem Alter und vergleichbarer Haushaltslage (vgl. Wanner/Fall 2011).

Zum zweiten wird ein Mangel an sozialen Kontakten nach einer Verwitwung in den aktuelleren Erhebungen weniger häufig angeführt, was mit einer in der Schweiz feststellbaren verbesserten sozialen Integration und verstärkten familialen wie außerfamilialen Sozialbeziehungen älterer Menschen verbunden sein dürfte (vgl. Höpflinger 2009b).

Während sich bei den wirtschaftlichen und sozialen Gegebenheiten (finanzielle Probleme, soziale Kontakte) bedeutsame Verbesserungen in der Lebenslage nach einer Verwitwung zeigen, ist dies bei den übrigen Items, die psychische Dimensionen (wie Einsamkeit, Lebenssinn) ansprechen, nicht der Fall. Bei diesen Items zeigen sich einige 
Schwankungen zwischen den Erhebungen, aber kein klarer, langfristiger Trend. Sinnzweifel werden je nach Erhebungsjahr von einem Fünftel bis einem Viertel der verwitweten Befragten angeführt. Auch der Zwang, nach einem Partnerverlust allein verantwortlich zu sein und alles selber zu machen, wird von manchen Befragten als schwerwiegende Herausforderung eingestuft. An erster Stelle der negativ erlebten Aspekte einer Verwitwung steht Einsamkeit. Im Periodenvergleich ergeben sich zwar statistisch signifikante Unterschiede, etwa zwischen 1979 und 1994 oder 1994 und 2011, aber im bivariaten Vergleich ist diesbezüglich kein eindeutiger Trend erkennbar.

Insgesamt findet die Ausgangsthese, dass periodenspezifische Veränderungen in der erlebten Bilanzierung einer Verwitwung primär wirtschaftliche und soziale Dimensionen betreffen, weniger jedoch psychische Dimensionen, eine gewisse empirische Bestätigung. Gleichzeitig wird deutlich, dass die stabilen Mittelwerte des (additiven) Gesamtindex wichtige Trendentwicklungen verbergen.

Der Mittelwertvergleich der Skala ,Bilanzierung empfundener Schwierigkeiten nach der Verwitwung' ließ keine deutlichen Unterschiede zwischen Männern und Frauen erkennen. Es gilt daher zu überprüfen, ob dies für alle Items gilt oder nicht. Tatsächlich ergeben sich bei zwei Bilanzierungsitems kontinuierliche Unterschiede zwischen männlichen und weiblichen Befragten. Dies verdeutlicht zusätzlich, dass die Gesamtskala - als additiver Summenindex - bedeutsame Differenzen verdeckt (vgl. Tabelle 3):

Zum ersten wird deutlich, dass Witwen durchgehend signifikant häufiger finanzielle Probleme nach einem Partnerverlust erwähnen als Witwer (auch wenn sich im Zeitverlauf die wirtschaftliche Lage für beide Gruppen verbessert hat). Dieses Ergebnis entspricht der allgemeinen Feststellung, dass Frauen in der Schweiz häufiger auf bedarfsorientierte $\mathrm{Zu}$ satzleistungen zu Altersrenten angewiesen sind als gleichaltrige Männer.

Tabelle 3: Bilanzierung der Verwitwung im Geschlechter- und Zeitvergleich (Prozentanteil jener, die schwerwiegende/große Probleme berichten)

\begin{tabular}{lccccccc}
\hline & \multicolumn{2}{c}{$\mathbf{1 9 7 9}$} & \multicolumn{2}{c}{$\mathbf{1 9 9 4}$} & \multicolumn{2}{c}{$\mathbf{2 0 1 1}$} & F \\
\hline Finanzielle Probleme & F & M & F & M & M \\
Mangel an sozialen Kontakten & 34 & $14^{*}$ & 27 & $6^{*}$ & 17 & $4^{*}$ \\
Einsamkeit & 23 & 30 & 14 & 23 & 13 & 19 \\
Sich daran gewöhnen, alles selber machen zu müssen & 42 & $53^{*}$ & 52 & 59 & 35 & 46 \\
Alleine Verantwortung übernehmen zu müssen & 26 & 27 & 33 & 30 & 23 & 31 \\
Dem Leben einen Sinn geben & 28 & $13^{*}$ & 35 & $19^{*}$ & 23 & 13 \\
\hline
\end{tabular}

F: Frauen, M: Männer

N 1979 (je nach Item) Frauen = 282-327, Männer = 104-112; N 1994 (je nach Item) Frauen = 301-318, Männer =104-113; N 2011 (je nach Item) Frauen = 202-206, Männer = 92-95.

* auf 1\% signifikante Unterschiede zwischen Männern und Frauen (Chi-Quadrat-Test).

Zum zweiten führen verwitwete Frauen häufiger an, dass die Übernahme der alleinigen Verantwortung nach dem Tod des Partners als Belastung erlebt wurde. Dies dürfte vor allem bei eher traditionell geführten Ehen (in denen der Ehemann Hauptträger ehelicher Entscheidungen war) der Fall sein. 2011 ist die Differenz - auch aufgrund der geringeren Fallzahlen - statistisch jedoch nicht mehr signifikant. Es ist durchaus denkbar, dass sich dank neuen Formen gleichberechtigter Partnerschaften mehr Frauen gewohnt sind, Ver- 
antwortung für sich und für andere zu übernehmen (wodurch sich die geschlechtsspezifischen Unterschiede bei diesem Bilanzierungsitem verwischt haben).

Bei den übrigen Items zeigen sich keine klaren, zeitübergreifenden Differenzen in den Antworten von Witwen und Witwern. Mangel an sozialen Kontakten und Einsamkeit als Folge einer Verwitwung werden zwar tendenziell von Männern etwas häufiger angeführt, aber die Differenzen sind hier (mit einer Ausnahme: Einsamkeit 1979) - im Gegensatz zu anderen Studien (Dykstra et al. 2004; Pinquart 2003) - statistisch nicht signifikant.

Die oft geäußerte These einer generell stärkeren psychischen Betroffenheit von Männern durch einen Partnerverlust wird nicht bestätigt, zumindest nicht was eine retrospektive Bilanzierung betrifft. Es ist allerdings zu berücksichtigen, dass in Querschnitterhebungen geschlechtsspezifische Unterschiede des Verwitwungserlebens durch Prozesse sozialer Selektion reduziert werden können; etwa wenn mehr Witwer nach einem Partnerverlust ein suizidales Verhalten bzw. eine erhöhte Mortalität aufweisen oder sie häufiger eine neue Partnerschaft eingehen als dies bei Witwen der Fall ist.

\section{Verwitwungsbilanz, subjektive Gesundheit und wahrgenommene soziale Unterstützung}

Aus der Forschungsliteratur ist bekannt, dass eine Verwitwung sich negativ auf die Gesundheit auswirken kann (vgl. Stroebe et al. 2007). Ebenso wird die These vertreten, dass eine erlebte soziale Unterstützung nach einem Partnerverlust die Bewältigung dieses lebenskritischen Ereignisses erleichtert. Es soll deshalb untersucht werden, inwiefern sich Zusammenhänge zwischen Verwitwungsbilanzierung, subjektiver Gesundheit und wahrgenommener sozialer Unterstützung im Zeitvergleich verändern oder stabil verbleiben. Da es sich hier um Querschnittsdaten handelt, lassen sich allerdings keine Analysen über eventuelle wechselseitige Einflüsse von subjektiver Gesundheit und Verwitwungsbilanzierung durchführen (sondern nur deskriptiv beschreiben und prüfen, ob sich (bivariate) Zusammenhänge periodenspezifisch verändert haben oder nicht).

Die bivariate Analyse (Tabelle 4) zeigt hochsignifikante Unterschiede der Verwitwungsbilanzierung je nach subjektiver Gesundheitseinschätzung - im Sinne einer positiven Assoziation zwischen positiver Verwitwungsbilanzierung und guter Gesundheit für die Jahre 1979 und 1994, aber nicht mehr für die Erhebung 2011.

Tabelle 4: Verwitwungsbilanzierung und subjektive Gesundheit im Zeitvergleich

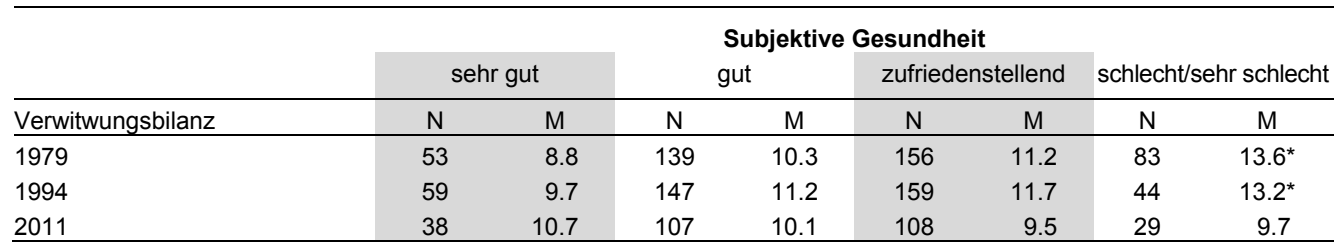

M: Mittelwerte des additiven Summenindexes, vgl. Tabelle 1.

* Unterschiede signifikant auf 1\%. F-Werte: 1979: 16.7; 1994: 6.5 2011: 0.94 (DF: 3). 
Dass sich die bivariaten Zusammenhänge zwischen Gesundheitserleben und Verwitwungsbilanzierung im Zeitverlauf abgeschwächt haben, wird auch darin deutlich, dass 1979 alle sechs Einzelitems signifikante Korrelationen mit der subjektiven Gesundheit aufwiesen. 1994 war dies nur noch für drei Einzelitems (Mangel an sozialen Kontakten, Einsamkeit, alles selber machen) der Fall und 2011 zeigt sich bei keinem der Items eine signifikante Korrelation zur subjektiven Gesundheit (vgl. Tabelle 5).

Tabelle 5: Zusammenhang zwischen angeführte Probleme nach Verwitwung und subjektiver Gesundheit im Zeitvergleich

\begin{tabular}{lccc}
\hline & \multicolumn{3}{c}{ Subjektive Gesundheit } \\
& 1979 & 1994 & 2011 \\
\hline N je nach Item & $431-446$ & $409-411$ & $282-294$ \\
Erlebte Probleme nach Verwitwung: & & & -.12 \\
Finanzielle Probleme & $-.22^{*}$ & -.11 & -.03 \\
Mangel an sozialen Kontakten & $-.26^{*}$ & $-.13^{*}$ & -.03 \\
Einsamkeit & $-.19^{*}$ & $-.18^{*}$ & -.11 \\
Sich daran gewöhnen, alles selber machen zu müssen & $-.25^{*}$ & $-.19^{*}$ & -.03 \\
Alleine Verantwortung übernehmen zu müssen & $-.22^{*}$ & -.10 & -.09 \\
Dem Leben einen Sinn geben & $-.11+$ & -.06 & \\
Gesamtskala Verwitwungsbilanz & & -.09 & -.09 \\
$\mathrm{~N}:$ & $-.31^{*}$ & 431 & 409 \\
\hline
\end{tabular}

*: signifikant auf $1 \%,+$ : signifikant auf $2 \%$.

Die festgestellte Verwischung der ursprünglich starken Beziehung zwischen Gesundheitsund Verwitwungserleben kann damit zusammenhängen, dass sich im Zeitverlauf - aufgrund besserer wirtschaftlicher und gesundheitspolitischer Rahmenbedingungen - die subjektive Gesundheit älterer verwitweter Frauen und Männer verbessert hat, speziell in der Schweiz, wo allgemein eine bedeutsame Ausdehnung der gesunden Lebensjahre stattgefunden hat (vgl. Höpflinger et al. 2011). 1979 stuften 19\% der befragten Witwen und Witwer ihre aktuelle Gesundheit als ,schlecht' bis ,sehr schlecht' ein und $45 \%$ als ,gut ${ }^{\star}$ bis ,sehr gut'. 2011 wurde die eigene Gesundheit nur noch von $10 \%$ der Befragten als ,schlecht' bis ,sehr schlecht' bewertet, wogegen 51\% ihre Gesundheit als ,gut' bis ,sehr gut $^{\star}$ einstuften. In jedem Fall weist der Zeitvergleich darauf hin, dass Zusammenhänge zwischen Partnerverlust und Gesundheit periodenspezifischen Veränderungen unterliegen. Dies impliziert, dass empirische Resultate aus früheren Studien nicht generell auf heutige Gesellschaften übertragen werden dürfen.

In allen drei Erhebungen wurde eine allgemeine, dichotom zu beantwortende Frage nach der Wahrnehmung erlebter sozialer Unterstützung nach dem Partnerverlust gestellt. Wird die wahrgenommene soziale Unterstützung mit der Bilanzierung der Verwitwung in Beziehung gesetzt (Tabelle 6), zeigt sich interessanterweise nicht der erwartete positive Zusammenhang, sondern - wenn überhaupt - ein negativer Zusammenhang sozialer Unterstützung in dem Sinn, dass Befragte, die eine Unterstützung erhielten, tendenziell mehr erlebte negative Herausforderungen anführen als Befragte, die mit keiner Unterstützung rechnen konnten. Die entsprechenden Unterschiede sind allerdings insgesamt gering, und nur in einer Erhebung (1994) statistisch signifikant. 
Tabelle 6: Verwitwungsbilanzierung und erlebte soziale Unterstützung nach Verwitwung im Geschlechter- und Zeitvergleich

\begin{tabular}{lcccccc}
\hline & \multicolumn{5}{c}{ Erlebte soziale Unterstützung nach Verwitwung } \\
& \multicolumn{3}{c}{ Alle } & \multicolumn{2}{c}{ Männer } & \multicolumn{2}{c}{ Frauen } \\
& Ja & Nein & Ja & Nein & Ja & Nein \\
\hline N 1979 & 282 & 145 & 72 & 37 & 210 & 108 \\
N 1994 & 269 & 117 & 63 & 38 & 206 & 79 \\
N 2011 & 204 & 68 & 63 & 23 & 142 & 45 \\
Mittelwerte & & & & & & \\
Verwitwungsbilanz 1979 & 11.5 & 10.5 & 11.2 & 9.5 & 11.6 & 10.9 \\
Verwitwungsbilanz 1994 & 12.2 & $10.8^{*}$ & 11.7 & 10.2 & 12.4 & 11.1 \\
Verwitwungsbilanz 2011 & 10.4 & 9.3 & 10.4 & 9.8 & 10.2 & 9.0 \\
\hline
\end{tabular}

*: signifikanter Unterschied nach erlebter sozialer Unterstützung auf 1\% (F-Test).

Die retrospektive Bilanzierung der Verwitwung scheint von der wahrgenommenen sozialen Unterstützung losgelöst zu sein, und die These, dass soziale Unterstützung das Erleben dieses kritischen Lebensereignisses erleichtert, erhält zumindest auf der Grundlage dieser Daten keine empirische Validierung und zwar weder bei Witwen noch bei Witwern (vgl. dazu auch Stroebe et al. 2005). Sichtbar wird im Zeitvergleich 1979-2011 allerdings, dass der Anteil von verwitweten Befragten, welche die Frage einer sozialen Unterstützung nach dem Partnerverlust positiv beantworteten, angestiegen ist (1979: 66\%, 1994: 70\%, 2011: 75\%). Soziale Unterstützung bei Verwitwung - auch dank erweiterten Angeboten der Altersberatung - hat zugenommen, aber ihre Auswirkung zumindest auf die retrospektive Bilanzierung der Herausforderungen, welche sich für den überlebenden Partner bzw. die überlebende Partnerin ergeben, bleibt unklar. Dies kann damit zusammenhängen, dass - wie in der Studie von Jung-Hwa Ha und Berit Ingersoll-Dayton (2011) deutlich wurde - weniger das Niveau der sozialen Unterstützung nach einer Verwitwung, sondern die Kongruenz zwischen gewünschter und erhaltener Unterstützung das Wohlbefinden bestimmt.

\section{Zusammenhänge mit chronologischem Alter, der Dauer seit Verwitwung und der Art des Partnerverlustes}

Im Folgenden wird überprüft, inwiefern weitere Variablen - soweit erfasst - mit der Verwitwungsbilanzierung in Zusammenhang stehen. Untersucht werden Zusammenhänge zwischen Verwitwungsbilanzierung und chronologischem Alter bei Befragung, Alter bei der Verwitwung und Dauer seit Verwitwung sowie mit der wahrgenommenen Vorhersehbarkeit des Todes des Ehepartners (unerwartet oder erwartet, z.B. nach langer Krankheit).

Das chronologische Alter scheint zu allen drei Zeitperioden für die Verwitwungsbilanzierung ohne große Bedeutung zu sein. Die linearen Korrelationen zwischen Verwitwungsbilanzierung und chronologischem Alter sind gering und nur 1979 leicht signifikant (1979: r: .10, sign. auf 5\%, N: 442, 1994: r: .08, nicht signifikant, N: 384, 2011: r: .02 , nicht signifikant, N: 282). Die Vermutung, dass eine Verwitwung im hohen Lebensalter besser bilanziert wird, findet keine Unterstützung, wie auch eine Sekundäranalyse des deutschen Alterssurveys 2002 belegt (vgl. Schmid 2010). 
Die Art und Weise des Partnerverlustes (plötzlich und unerwartet oder voraussehbar aufgrund einer Krankheit) wurde 1979 nicht erfragt, jedoch 1994 und 2011. Die These, dass ein unerwarteter Partnerverlust negativer erlebt wird als ein Partnerverlust nach einer Erkrankung wird umstritten diskutiert und ist empirisch nicht eindeutig belegt (vgl. Carr et al. 2001). Bei einer Verwitwung vor allem im höheren Lebensalter ist zu beachten, „dass dem Partnerverlust häufig lang anhaltende Gesundheitsprobleme des Ehepartners und eine damit verbundene belastende Phase des Pflegens vorausgeht." (Boerner 2012: 233). In unseren Daten zeigt sich, dass ein plötzlicher, unerwarteter Tod retrospektiv leicht negativer bilanziert wird als ein Partnerverlust, der etwa aufgrund einer Krankheit erwartet wurde (vgl. Tabelle 7). Allerdings sind die Unterschiede nicht besonders ausgeprägt und bezüglich der Skala der Verwitwungsbilanzierung nur auf dem 5\%-Niveau statistisch signifikant. 1994 (aber nicht mehr 2011) wurden Einsamkeitsgefühle und sich daran gewöhnen, alles selber machen zu müssen, bei einem unerwarteten Tod signifikant stärker gewichtet. Aber insgesamt ergeben sich zwischen der retrospektiven Bilanzierung einer Verwitwung und der Art des Partnerverlustes (erwartet oder unerwartet) höchstens schwache Zusammenhänge (zumindest auf der Grundlage standardisierter Erhebungen).

Tabelle 7: Empfundene Schwierigkeiten nach der Verwitwung nach Vorhersehbarkeit des Partnerverlustes

\begin{tabular}{|c|c|c|c|c|c|}
\hline & \multirow[b]{2}{*}{ Tod } & \multicolumn{2}{|c|}{1994} & \multicolumn{2}{|c|}{2011} \\
\hline & & erwartet & unerwartet & erwartet & unerwartet \\
\hline Erlebte Probleme nach Verwitwung: & $\mathrm{N}$ & 253 & 161 & 169 & 126 \\
\hline Finanzielle Probleme & & 1.7 & 1.6 & 1.3 & 1.5 \\
\hline Mangel an sozialen Kontakten & & 1.5 & 1.6 & 1.4 & 1.6 \\
\hline Einsamkeit & & 2.3 & $2.6^{*}$ & 2.0 & 2.2 \\
\hline Sich daran gewöhnen, alles selber machen zu müssen & & 1.8 & $2.1^{*}$ & 1.7 & 1.8 \\
\hline Alleine Verantwortung übernehmen zu müssen & & 1.8 & 1.9 & 1.5 & $1.8+$ \\
\hline Dem Leben einen Sinn geben & & 1.9 & 1.9 & 1.6 & 1.7 \\
\hline Gesamtskala & $\mathrm{N}$ & 231 & 153 & 159 & 121 \\
\hline Verwitwungsbilanz & & 10.9 & $11.8+$ & 9.5 & $10.4+$ \\
\hline
\end{tabular}

*: sign. auf $1 \%,+$ : sign. auf 5\% (F-Test)

Das Alter bei der Verwitwung und die Zeitdauer seit der Verwitwung sind eng korreliert. $\mathrm{Zu}$ allen Zeitpunkten ergeben sich Interkorrelationen von $\mathrm{r}=-.80$ und mehr. Damit erschweren Probleme der Multikollinearität eine eindeutige Interpretation der entsprechenden Einzelvariablen.

Die Beziehungen zwischen dem Alter bei der Verwitwung bzw. der Zeitdauer seit Verwitwung und der Skala der Gesamtbilanzierung einer Verwitwung sind übers Ganze betrachtet relativ schwach und statistisch signifikante (lineare) Beziehungen finden sich nur bei Witwen (vgl. Tabelle 8). Die Vermutung, dass sich die Gesamtbilanzierung einer Verwitwung etwa nach einer längeren Phase der Verwitwung in Richtung einer weniger negativen Bewertung - im Sinne einer möglicherweise auch resignativen Anpassung an die neue Situation - verändert, findet keine Bestätigung. Umgekehrt kann dies bedeuten, dass die retrospektive Bilanzierung von der Dauer seit der Verwitwung relativ unabhängig ist, was auf eine lebenszeitbezogene Stabilität einer Verwitwungsbilanzierung deuten könnte. 
Tabelle 8: Zusammenhang zwischen Alter bei Verwitwung und Dauer seit Verwitwung mit Verwitwungsbilanzierung

\begin{tabular}{lcccccc}
\hline & \multicolumn{3}{c}{ Alter bei Verwitwung } & \multicolumn{3}{c}{ Dauer seit Verwitwung } \\
& 1979 & 1994 & 2011 & 1979 & 1994 & 2011 \\
\hline Total (N) & 396 & 416 & 281 & 396 & 416 & 283 \\
Skala Gesamtbilanzierung & -.08 & $-.15^{*}$ & -.06 & .02 & .09 & .10 \\
Finanzielle Probleme & $-.31^{*}$ & $-.31^{*}$ & $-.21^{*}$ & $.28^{*}$ & $.33^{*}$ & $.26^{*}$ \\
Allein Verantwortung übernehmen & $-.13^{*}$ & $-.19^{*}$ & -.08 & .07 & $.14^{*}$ & $.14+$ \\
\hline Männer (n) & 107 & 112 & 87 & 107 & 112 & 89 \\
Skala Gesamtbilanzierung & -.04 & -.10 & -.14 & .20 & .02 & .02 \\
Finanzielle Probleme & -.07 & $-.27^{*}$ & -.16 & .11 & .17 & .16 \\
Allein Verantwortung übernehmen & -.01 & -.10 & -.03 & .11 & .01 & .03 \\
\hline Frauen (n) & 289 & 311 & 194 & 289 & 311 & 194 \\
Skala Gesamtbilanzierung & -.11 & $-.14+$ & $-.15+$ & .08 & .09 & $.16+$ \\
Finanzielle Probleme & $-.33^{*}$ & $-.33^{*}$ & $-.26^{*}$ & $.35^{*}$ & $.31^{*}$ & $.29^{*}$ \\
Allein Verantwortung übernehmen & $-.13+$ & $-.18^{*}$ & -.13 & .09 & $.14^{*}$ & $.18^{*}$ \\
\hline
\end{tabular}

* signifikant auf $1 \%,+$ signifikant auf 5\%

Bei den übrigen vier Einzelitems: keine signifikanten Korrelationen

Nur bei zwei der sechs Einzelitems ergeben sich periodenübergreifende Korrelationen, wobei diese Beziehungen primär bei verwitweten Frauen auftreten:

Zum ersten werden die negativen finanziellen Folgen einer Verwitwung - von verwitweten Frauen - umso stärker erlebt, je früher eine Verwitwung erfolgte und je länger der Status der Verwitwung anhält. Dies hängt damit zusammen, dass negative finanzielle Folgen einer Verwitwung (bei Frauen) vor allem längerfristig sichtbar werden, wenn etwa die Konsequenzen reduzierter Altersrenten deutlicher werden. Die entsprechenden Korrelationen sind zu allen drei Zeitpunkten statistisch signifikant.

Zum zweiten ist die negative Beurteilung der alleinigen Verantwortung etwas ausgeprägter, wenn eine Verwitwung relativ früh auftritt (negative Korrelation mit dem Alter bei Verwitwung). Mit steigender Dauer einer Verwitwung wird das Problem der alleinigen Verantwortung stärker hervorgehoben (wobei die hohe Kollinearität zwischen Dauer seit Verwitwung und Alter bei Verwitwung verhindert, den Effekt des Alters bei Verwitwung und den Effekt der Dauer seit Verwitwung zu differenzieren). ${ }^{6}$ Auch in dieser Hinsicht treten die entsprechenden Korrelationen hauptsächlich bei verwitweten Frauen auf.

Insgesamt gesehen beeinflussen die einbezogenen zusätzlichen Variablen (Alter bei Verwitwung bzw. Dauer seit Verwitwung) die Gesamtbilanzierung einer Verwitwung so wie sie erfasst wurde - wenig. Sofern Alters- oder Dauereffekte sichtbar werden, betrifft dies vor allem Frauen bezüglich finanzieller Folgen und der Wahrnehmung alleiniger Verantwortlichkeit.

Im Abschluss soll die bivariate Analyse durch eine multivariate Analyse ergänzt werden, wobei neben den bisher eingeführten Variablen auch zwei sozio-strukturelle Variablen einbezogen werden (Bildungsstatus, relatives Haushaltseinkommen). Aus entwick-

6 Dazu müssten die Fallzahlen wesentlich erhöht werden, um alle Kombinationen in genügender Zahl aufzuweisen. Auch die Tatsache, dass nur verwitwete Personen im Alter 65+ einbezogen sind, erschwert eine klare Interpretation der Folgen einer frühen Verwitwung. 
lungspsychologischer Sicht ist eine multivariate Analyse, welche keine oder kaum Zusammenhänge zwischen Verwitwungsbilanzierung und sozialen Variablen aufweist, ideal, weil damit die sozial übergreifende Validität der verwendeten Skala unterstützt wird. Aus soziologischer Sicht wären klare Zusammenhänge zwischen Verwitwungserleben und sozialen Variablen ideal, weil Verwitwung damit als von der sozialen Lebenslage bestimmtes kritisches Lebensereignis interpretiert werden kann (und nicht allein als psychologisches Einzelereignis).

Die Ergebnisse der durchgeführten multivariaten Varianzanalyse unterstützen - was die Gesamtskala der Verwitwungsbilanzierung betrifft - eher eine psychologische als eine soziologische Interpretation. Soziale Variablen (Bildungsstatus, relatives Einkommen, aber auch Geschlecht, Alter, Dauer der Verwitwung, Art des Todes des Partners bzw. der Partnerin) sind von überraschend geringer Bedeutung. Signifikante Zusammenhänge ergeben sich einzig mit zwei Variablen, und dies auch nur für die ersten zwei Zeitperioden (1979, 1994): subjektive Gesundheit und erhaltene Unterstützung. In der letzten Studie (2011) verlieren auch diese Zusammenhänge an Bedeutung (was die Ergebnisse der vorgängigen bivariaten Analyse unterstützt).

Tabelle 9: Multivariate Analyse zur Verwitwungsbilanzierung

\begin{tabular}{|c|c|c|c|c|c|c|c|c|c|}
\hline & \multicolumn{9}{|c|}{ Abhängige Variable: Skala ,Verwitwungsbilanz‘ } \\
\hline & \multicolumn{3}{|c|}{1979} & \multicolumn{3}{|c|}{1994} & \multicolumn{3}{|c|}{2011} \\
\hline & df & $\mathrm{F}$ & $\mathrm{p}$ & $\mathrm{df}$ & $\mathrm{F}$ & $\mathrm{p}$ & $\mathrm{df}$ & $\mathrm{F}$ & $\mathrm{p}$ \\
\hline Korrigiertes Modell & 11 & 6.0 & .000 & 11 & 3.5 & .000 & 11 & 1.2 & .290 \\
\hline Konstanter Term & 1 & 34.0 & .000 & 1 & 31.9 & .001 & 1 & 92.7 & .000 \\
\hline \multicolumn{10}{|l|}{ Kovariate: } \\
\hline Chronologisches Alter & 1 & 3.3 & .071 & 1 & 0.6 & .448 & 1 & 0.4 & .504 \\
\hline Jahre seit Partnerverlust & 1 & 0.2 & .648 & 1 & 1.4 & .238 & 1 & 1.6 & .202 \\
\hline \multicolumn{10}{|l|}{ Unabhängige Variablen: } \\
\hline Bildungsstatus & 2 & 0.2 & .847 & 2 & 0.3 & .772 & 2 & 0.2 & .853 \\
\hline Relatives Einkommen & 1 & 1.7 & .190 & 1 & 0.9 & .345 & 1 & 1.5 & .217 \\
\hline Geschlecht & 1 & 0.5 & .471 & 1 & 0.6 & .436 & 1 & 1.7 & .188 \\
\hline Erhaltene Unterstützung & 1 & 4.7 & .010 & 1 & 8.9 & .003 & 1 & 1.5 & .222 \\
\hline Subjektive Gesundheit & 3 & 14.5 & .000 & 3 & 6.0 & .001 & 3 & 0.7 & .572 \\
\hline Art des Todes des Partners & & & & 1 & 1.4 & .232 & 1 & 3.5 & .064 \\
\hline$R^{2}$ & & & .150 & & & .131 & & & .06 \\
\hline$R^{2}$ korrigiert & & & .125 & & & .094 & & & .01 \\
\hline$N:$ & & & 388 & & & 269 & & & 222 \\
\hline
\end{tabular}

Methode: Multivariate Varianzanalyse (general linear model)

Bildungsstatus: dreistufig: a) nur obligatorische Schule, ohne weiterführende berufliche Ausbildung, b) berufliche Fachausbildung (Lehre u.a.), c) Tertiäre Ausbildung (Fachhochschule, Universität).

Relatives Einkommen: zweistufig: a) Haushaltseinkommen geringer als Medianwert, b) Haushaltseinkommen höher als Medianwert.

Erhaltene Unterstützung nach Verwitwung: ja versus nein

Subjektive Gesundheit: sehr gut, gut, mittelmäßig, schlecht/sehr schlecht

Art des Todes des Partners: unerwartet versus erwartet, nach langer Krankheit. 


\section{Zusammenfassung und Schlussfolgerungen}

Der Vergleich der subjektiven Gesamtbilanzierung einer Verwitwung über eine Zeitperiode von mehr als dreißig Jahren verdeutlicht eine erstaunliche Konstanz. Die verwendete Skala ,Bilanzierung empfundener Schwierigkeiten nach der Verwitwung' weist zudem eine hohe, zeitübergreifende Konstruktreliabilität auf. Die Analyseergebnisse deuten darauf hin, dass Verwitwung zumindest retrospektiv unabhängig von den Zeitumständen ähnliche Herausforderungen impliziert. Diese Interpretation wird dadurch gestützt, dass die berücksichtigten intervenierenden Variablen - wie Geschlecht, aktuelles Alter, Alter bei Verwitwung bzw. Dauer seit Verwitwung - die retrospektive Bilanzierung einer Verwitwung wenig beeinflussen. Die Resultate stützen einen mehr psychologischen Interpretationsansatz, der davon ausgeht, dass Verwitwung primär ein individuelles und individuell zu bewältigendes kritisches Lebensereignis darstellt, welches unabhängig von gesellschaftlichen Rahmenbedingungen als psychisch herausfordernd erfahren wird. Unterschiede in der Bewältigung einer Verwitwung scheinen in diesem Fall primär durch individuelle Ressourcen und Copingstrategien bestimmt zu sein und weniger durch soziale, familiale oder wirtschaftliche Umgebungsfaktoren.

Die Gesamtskala verdeckt allerdings Veränderungen in einigen bedeutsamen Einzelaspekten: Erstens werden finanzielle Probleme einer Verwitwung in den neueren Erhebungen signifikant seltener angeführt, wobei gleichzeitig Witwen durchgehend signifikant häufiger finanzielle Probleme nach einem Partnerverlust anführen als Witwer. Zweitens wird ein erlebter Mangel an sozialen Kontakten nach einer Verwitwung in neuerer Zeit weniger häufig angeführt. Erlebte soziale Unterstützung nach einer Verwitwung wird im Zeitverlauf häufiger erwähnt, aber die Beziehungen zwischen erlebter sozialer Unterstützung und Verwitwungsbilanzierung sind durchgehend schwach. Zusätzlich wird sichtbar, dass sich ursprünglich hochsignifikante Unterschiede der Verwitwungsbilanzierung je nach subjektiver Gesundheitseinschätzung in der neuesten Erhebung 2011 aufgelöst haben, was mit einer allgemein verbesserten Gesundheit älterer Frauen und Männer in Verbindung steht. Zusammenhänge zwischen Partnerverlust und Gesundheit unterliegen damit relevanten periodenspezifischen Veränderungen.

Kein klarer Zeittrend ergibt sich auf der anderen Seite bei Items, die mehr psychische Aspekte ansprechen. So wird die Notwendigkeit nach einem Partnerverlust, allein verantwortlich zu sein oder dem Leben einen neuen Sinn zu geben, durchgehend von einer beträchtlichen Minderheit als bedeutsame Herausforderung erlebt. $\mathrm{Zu}$ allen drei Erhebungszeitpunkten stehen Einsamkeitsgefühle an erster Stelle der negativ erlebten Verwitwungsfolgen. Einsamkeit als Folge einer Verwitwung wird zwar von Männern etwas öfter angeführt, aber im Gegensatz zu früheren Studien (Dykstra et al. 2004; Pinquart 2003) sind die diesbezüglichen geschlechtsspezifischen Unterschiede nicht durchgängig signifikant; möglicherweise in unseren Daten auch als Folge sozialer Selektionseffekte, die bei Querschnittserhebungen nicht zu kontrollieren sind (etwa wenn verwitwete Männer Einsamkeit nach Partnerverlust durch eine neue Partnerbeziehung kompensieren).

Insgesamt findet die Ausgangsthese, dass periodenspezifische Veränderungen in den erlebten Folgen einer Verwitwung primär wirtschaftliche und soziale Dimensionen betreffen, weniger jedoch psychische Dimensionen, eine empirische Bestätigung. Die äußeren Rahmenbedingungen nach einer Verwitwung (finanzielle Lage, soziale Kontakte und so- 
ziale Unterstützung, Gesundheit) haben sich teilweise substanziell verbessert, aber dies hat sich kaum auf die erlebten psychischen Herausforderungen (neuen Lebenssinn finden; sich daran gewöhnen, alles allein zu erledigen; Einsamkeitsgefühle) von Witwen und Witwern ausgewirkt. Verwitwung bleibt auch bei günstigen Sozialbedingungen ein kritisches Lebensereignis, das in starkem Maße individuell zu bewältigen ist (Perrig-Chiello 2007). Soziologische Lebenslagenkonzepte stoßen bei diesem kritischen Lebensereignis auf ihre Grenzen und ohne Berücksichtigung individualpsychologischer Ansätze lässt sich Verwitwung nicht verstehen.

\section{Literatur}

Bennett, K. M. (1997). Widowhood in elderly women: The medium- and long-term effects on mental and physical health. Mortality, 2, 2, S. 137-148.

Bennett, K. M. (2005). Psychological wellbeing in later life: The longitudinal effects of marriage, widowhood and marital status change. International Journal of Geriatric Psychiatry, 20, 3, S. 280-284.

Bennett, K. M. (2008). Widowhood. In: Carr, D. (Hrsg.), Encyclopedia of the life course and human development. Farmington Hills, MI: Gale Publ., S. 438-444.

Bennett, K. M. \& Soulsby, L. K. (2012). Wellbeing in bereavement and widowhood. Illness, Crisis and Loss, 20, 4, S. 321-337.

Berg, A. I., Hassing, L. B., Mcclearn, G. E. \& Johansson, B. (2006). What matters for life satisfaction in the oldest-old? Aging and Mental Health, 10, 3, S. 257-264.

Boerner, K. (2012). Umgang mit Verwitwung. In: Wahl, H.-W., Tesch-Römer, C. \& Philipp-Ziegelmann, J. (Hrsg.), Angewandte Gerontologie. Interventionen für ein gutes Altern in 100 Schlüsselbegriffen. Stuttgart: Kohlhammer, S. 230-235.

Bonanno, G. A., Wortman, C. B. \& Nesse, R. M. (2004). Prospective patterns of resilience and maladjustment during widowhood. Psychology and Aging, 19, 2, S. 260-271.

Burkauser, R. V., Giles, P., Lillard, D. R. \& Schwarze, J. (2005). Until death us do part: an analysis of the economic well-being of widows in four countries. Journal of Gerontology: Social Sciences, 60, S. 238-246.

Carr, D. (2004). The desire to date and remarry among older widows and widowers. Journal of Marriage and Family, 66, S. 1051-1068.

Carr, D., House, J. S., Wortman, C., Neese, R. \& Kessler, R. C. (2001). Psychological adjustment to sudden and anticipated spousal loss among older widowed persons. Journal of Gerontology: Social Sciences, 56B, 4, S. 237-248.

Clark, A. E., Diener, E., Georgellis, Y. \& Lucas, R. E. (2008). Lags and leads in life satisfaction: A test of the baseline hypothesis. Economic Journal, 118, 529, S. 222-243.

Delbès, C. \& Gaymu, J. (2002). The shock of widowhood on the eve of old age: Male and female experiences. Population (English Edition), 57, 6, S. 885-913.

Dykstra, P. A. \& de Jong Gierveld, J. (2004). Gender and marital-history differences in emotional and social loneliness among Dutch older adults. Canadian Journal on Aging, 23, 2, S. 141-155.

Gorlé, S. \& van den Bosch, K. (2008). Inequality, life-course transitions, and income position. In: Börsch-Supan, A., Brugiavini, A., Jüges, H. et al. (Hrsg.), First results from the Survey of Health, Ageing and Retirement in Europe (2004-2007). Starting the longitudinal dimension. Mannheim: Mannheim Research Institute for the Economics of Aging, S. 297-305.

GUGRISPA (Groupe Universitaire Genevois) (1983). Vieillesses. Situations, itinéraires et modes de vie des personnes âgées aujourd'hui. Saint-Saphorin: Georgi.

Ha, J.-H. \& Ingersoll-Dayton, B. (2011). Moderators in the relationship between social contact and psychological distress among widowed adults. Aging and Mental Health, 15, 3, S. 354-363.

Heiniger, M. (2013). Die Zivilstandsstruktur der ständigen Wohnbevölkerung, Demos, Newsletter. Informationen aus der Demografie, Nr. 1/2013. Bern: Bundesamt für Statistik, S. 2-6. 
Hollstein, B. (2002). Soziale Netzwerke nach der Verwitwung. Eine Rekonstruktion der Veränderungen informeller Beziehungen. Opladen: Leske + Budrich.

Höpflinger, F. (2009a) Einblicke und Ausblicke zum Wohnen im Alter. Age Report 2009. Zürich: Seismo.

Höpflinger, F. (2009b). Sozialgerontologie: Alter im gesellschaftlichen Wandel und neue soziale Normvorstellungen zu späteren Lebensjahren. In: Klie, T. Kumlehn M. \& Kunz, R. (Hrsg.), Praktische Theologie des Alterns. Berlin: Walter de Gruyter, S. 55-73.

Höpflinger, F. (2011). Die neue Generation der ,jungen Alten'. Befindlichkeit und Werthaltungen in mitteleuropäischen Ländern. Psychotherapie im Alter, 2, 8, S. 155-166.

Höpflinger, F., Bayer-Oglesby, L. \& Zumbrunn, A. (2011). Pflegebedürftigkeit und Langzeitpflege im Alter. Aktualisierte Szenarien für die Schweiz. Bern: Huber.

Itzhar-Naborro, Z. \& Smoski, M. J. (2012). A review of theoretical and empirical perspectives on marital satisfaction and bereavement outcomes: Implications for working with older adults. Clinical Gerontologist: The Journal of Aging and Mental Health, 35, 3, S. 257-269.

Jagger, C., Weston, C., Cambois, E., Van Oyen, H., Nusselder, W., Doblhammer, G. \& Team, E. (2011). Inequalities in health expectancies at older ages in the European Union: findings from the Survey of Health and Retirement in Europe (SHARE). Journal of Epidemiology and Community Health, 65, 11, S. 1030-1035.

Jin, L. \& Chrisatakis, N. A. (2009). Investigating the mechanism of marital mortality reduction: The transition to widowhood and quality of health care. Demography 46, 3, S. 605-625.

Koren, C. \& Lowenstein, A. (2008). Late-life widowhood and meaning in life. Ageing International, 32, 2, S. 140-155.

Lalive d'Epinay, C., Bickel, J.-F., Maystre, C. \& Vollenwyder, N. (2000). Vieillesses au fil du temps 1979-1994. Une révolution tranquille. Lausanne: Réalités Sociales.

Lee, G. R. \& DeMaris, A. (2007). Widowhood, gender, and depression - A longitudinal analysis. Research on Aging, 29, 1, S. 56-72.

Lee, G. R., DeMaris, A., Bavin, S. \& Sullivan, R. (2001). Gender differences in the depressive effect of widowhood in later life. Journal of Gerontology: Social Sciences, 56, 1, S. 56-61.

Lucas, R. E., Clark, A. E., Georgellis, Y. \& Diener, E. (2003). Reexamining adaptation and the set point model of happiness: Reactions to changes in marital status. Journal of Personality and Social Psychology, 84, 3, S. 527-539.

Moser, P. (2006) Einkommen und Vermögen der Generationen im Lebenszyklus. Eine QuerschnittsKohortenanalyse der Zürcher Staatssteuerdaten 1991-2003. Zürich: Statistisches Amt des Kantons Zürich (statistik.info 1/2006).

Norris, F. H. \& Murrell, S. A. (1990). Social support, life events, and Stress as modifiers of adjustment to bereavement in older adults. Psychology \& Aging, 5, 3, S. 429-436.

Perkins, H. W., \& Harris, L. B. (1990). Familial bereavement and health in adult life course perspective. Journal of Marriage and the Family, 52, 1, S. 233-241.

Perrig-Chiello, P. (2007). Alter, Gesundheit und Geschlecht. Leading Opinions in Neurologie und Psychiatrie, 3, S. 24-26.

Perrig-Chiello, P. \& Hutchison, S. (2010). Health and well-being in old age - The pertinence of a gender-mainstreaming approach in research. Gerontology, 56, 2, S. 208-213.

Pinquart, M. (2003). Loneliness in married, widowed, divorced, and never-married older adults. Journal of Social and Personal Relationships, 20, 1, S. 31-53.

Schaan, B. (2009). Verwitwung, Geschlecht und Depression im höheren Lebensalter. In: Börsch-Supan, A., Karsten, H. Hendrik, J. \& Schröder, M. (Hrsg.), 50plus in Deutschland und Europa: Ergebnisse des Survey of Health, Ageing and Retirement in Europe. Wiesbaden: VS Verlag, S. 115-131.

Schmid, S. K. E. (2010). Frauen nach der Verwitwung. Soziale Netzwerke als Hilfestellung für Verwitwete. Wien: Universität Wien (Diplomarbeit).

Stroebe, M., Stroebe, W. \& Schut, H. (2001). Gender differences in adjustment to bereavement: An empirical and theoretical review. Review of General Psychology, 5, S. 62-83.

Stroebe, M., Schut, H. \& Stroebe, W. (2007). Health outcomes of bereavement. The Lancet, 370, 9603, S. 1960-1973. 
Stroebe, W., Zech, E., Stroebe, M. \& Abakoumkin, G. (2005). Does social support help in bereavement? Journal of Social and Clinical Psychology, 24, 7, S. 1030-1050.

Wanner, P. \& Fall, S. (2011). La situation économique des veuves et des veufs. Genève: Laboratoire démographique de l'Université de Genève.

van Grootheest, D. S., Beekman, A. T. F., Broese van Groenou, M. I. \& Deeg, D. J. H. (1999). Sex differences in depression after widowhood. Do men suffer more? Social Psychiatry and Psychiatric Epidemiology, 34, 7, S. 391-398.

Eingereicht am/Submitted on: 29.11.2012

Angenommen am/Accepted on: 17.06.2013

Anschriften des Autors und der Autorinnen/Addresses of the authors:

Prof. Dr. François Höpflinger

Soziologisches Institut der Universität Zürich

Andreasstrasse 15

8050 Zürich-Oerlikon

Schweiz/Switzerland

Stefanie Spahni, M. Sc.

Prof. Dr. Pasqualina Perrig-Chiello

Universität Bern

Institut für Psychologie

Fabrikstrasse 8

3012 Bern

Schweiz/Switzerland

E-Mail: hoepflinger@bluemail.ch stefanie.spahni@psy.unibe.ch pasqualina.perrigchiello@psy.unibe.ch 